\title{
Regenerative Endodontic Procedure as a Retreatment Alternative for Permanent Maxillary Central Incisors with Esthetic Smile Correction
}

\section{Rishi Tyagi, Namita Kalra, Amit Khatri, Puja Sabherwal*, Khadeeja Kulood and Padma Yangdol}

Department of Paedodontics and Preventive Dentistry, University College of Medical

Sciences, Guru Teg Bahadur Hospital, Dilshad Garden, Delhi, India

*Corresponding Author: Puja Sabherwal, Department of Paedodontics and

Preventive Dentistry, University College of Medical Sciences, Guru Teg

Bahadur Hospital, Dilshad Garden, Delhi, India.
Received: September 14, 2021

Published: October 19, 2021

(C) All rights are reserved by Sabherwal., et al.

\begin{abstract}
Regenerative Endodontic Procedures (REP) provide modern dentistry with a biological minimally invasive option to manage young and necrosed teeth. Now more than ever before, the biological and intrinsic physiological healing response of the human body is being applied to a multitude of medical and dental procedures. REP emulates additional benefits over the traditional procedures leading to increased root length/ width with optimum strength of the pulp-dentin-cementum complex. Two methodologies of the REP protocol have been employed in the present case report as a retreatment modality for permanent maxillary central incisors. The teeth had a long-term history of traumatic dental injuries and successively failed root canal treatments suggestive of poor prognosis. Hence, the clinical and radiographic success for the 11-year-old boy with two years of follow-up alongside esthetic smile correction using an inter-disciplinary approach proved to be of immense value. These outcomes led to an overall improvement in the quality of life with extended preservation of the natural teeth until further prosthesis may be feasible.
\end{abstract}

Keywords: Regenerative Endodontic Procedures; Minimally Invasive; Biological; Esthetic; Inter-disciplinary Dentistry

\section{Abbreviations}

REP: Regenerative Endodontic Procedure; EDTA: Ethylene Diamine Tetra-Acetic Acid; GIC: Glass Ionomer Cement; MTA: Mineral Trioxide Aggregate

\section{Introduction}

Regenerative endodontic procedure (REP) is a biologicallybased treatment modality that induces regeneration of pulp dentin complex thereby leading to normal thickening of the root via stimulation of stem cells [1]. Historically, G.L Feldman (1932) showed the first evidence of regeneration in dentistry and NygaardOstby (1971) conducted a neoteric study that noted induction of bleeding in a necrotic tooth which led to the reconstitution of the immature apex [2]. Windley (2005) proposed criteria for successful regeneration that include canal disinfection, scaffold placement for tissue growth and a hermetic seal [3]. At the molecular level, the success relies on the interplay between stem cells, scaffolds and signalling molecules constitutively called classical tissue engineering triad [1].

The present case report depicts two methodologies for regenerative rehabilitation of immature permanent maxillary central incisors with a history of traumatic dental injury and failed root canal treatment two years ago. The case was followed up over a period of 24 months elucidating continued root formation with the resolution of clinical and radiographical signs and symptoms.

\section{Case Report}

A 12-year-old boy reported to the Department of Pedodontics for the chief complaint of pain and swelling in the upper front tooth region since one year. The pain was of mild-moderate intensity, 
throbbing nature, radiating to the jaw and nasal region, accompanied by yellow-white pus discharge with exacerbation present nocturnally and while eating; relieved only mildly with over-thecounter pain medication. The mother's statement recorded a history of traumatic dental injury to the same region two years ago for which the child underwent treatment at various local dental clinics.

The case history records revealed no significant family, medical, drug or allergic conditions. The clinical and radiographic examination recorded the following findings -Ellis Class III fracture with Chronic Alveolar Abscess, failed root canal treatment and open apices-11, 21, Grade I mobility-21, congenitally absent-22 (Figure $1 \mathrm{a}, \mathrm{b})$. The radiograph was taken using the long cone parallel technique using $24 \times 40 \mathrm{~mm}$ size film (Kodak Ultraspeed size II Dental Film, Eastman Kodak), parallel to the long axis of the tooth and perpendicular to the X-ray central cone.
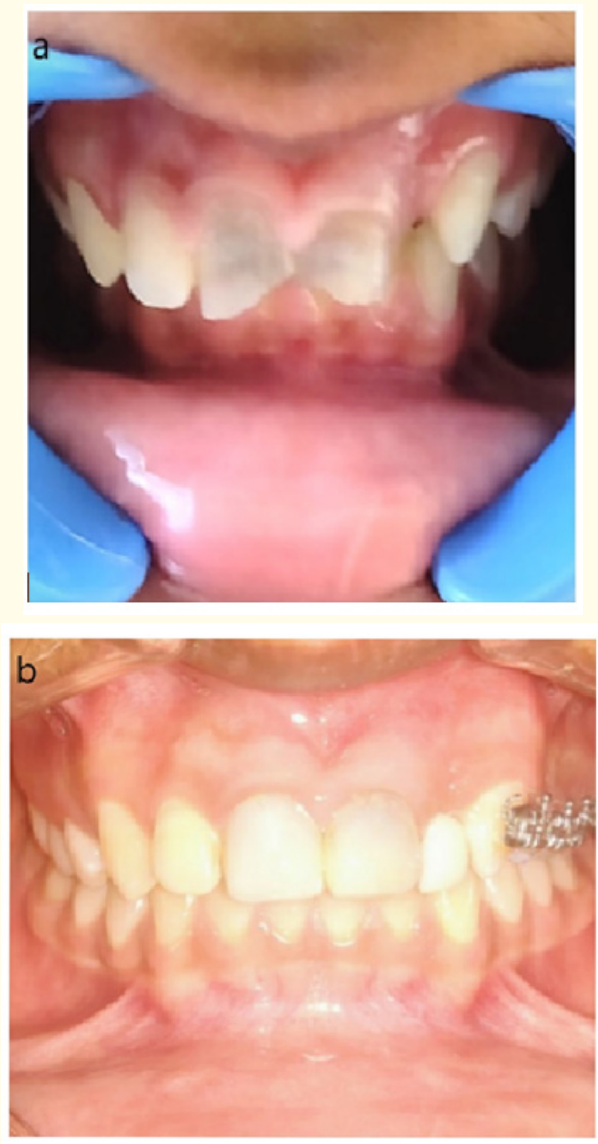

Figure 1: Preoperative buccal view.

The prognosis and age-related treatment options were explained to the patient's parents. The importance of routine follow-ups and compliance was positively reinforced and informed consent as well as assent was obtained. The treatment was planned under three phases for ease of operator and patient compliance.

During the phase, I, faulty root canal filling material was removed (Figure $2 \mathrm{a}, \mathrm{b}$ ) followed by canal disinfection of 11 using 3-Mix Antibiotic Paste (1:1:1) comprising of Ciprofloxacin (200mg), Metronidazole (400mg) and Minocycline(100mg) mixed with a concentration of $0.01-0.1 \mathrm{mg} / \mathrm{ml}$ (Figure 2c) [4]. The disinfection of 21 was done using Curcumin longa (300mg) and piperine (Turmix, Sanat Products Ltd, India) with propylene glycol (1:1 ratio) (Figure 2d) [5]. Post four weeks of disinfection, the patient reported resolution of clinical signs and symptoms. Subsequently, chemo-mechanical preparation for 11 was done using irrigation with $1 \%$ sodium hypochlorite $(20 \mathrm{~mL})$, saline $(20 \mathrm{~mL}), 17 \%$ Ethylene Diamine TetraAcetic acid (EDTA) $(20 \mathrm{~mL})$ respectively followed by induction of bleeding using EDTA soaked \#20K file (Dentsply Maillefer, Ballaigues, Switzerland) by probing $2 \mathrm{~mm}$ overworking length. The clot was allowed to fill the canal up to the cementoenamel junction and gel foam (FLOSEAL Hemostatic Matrix, Baxter HC, USA) was used as a scaffold. The coronal part of the canal was sealed with mineral trioxide aggregate (Dentsply Tulsa Dental, USA) $2 \mathrm{~mm}$ apical to cementoenamel junction followed by Glass Ionomer Cement (GIC) (GC, Tokyo, Japan). The regenerative approach for 21 included the above-mentioned irrigation protocol followed by placement of Choukron's Platelet Rich Fibrin (PRF) prepared by centrifugation of the patient's blood (3000 rpm; $10 \mathrm{~min}$ ) (Figure 2e,f). PRF was drawn into a thin membrane using two glass slabs and inserted into the canal using a sterile tweezer and plugger. The coronal third was sealed using Mineral Trioxide Aggregate(MTA) and GIC was placed as above [6].
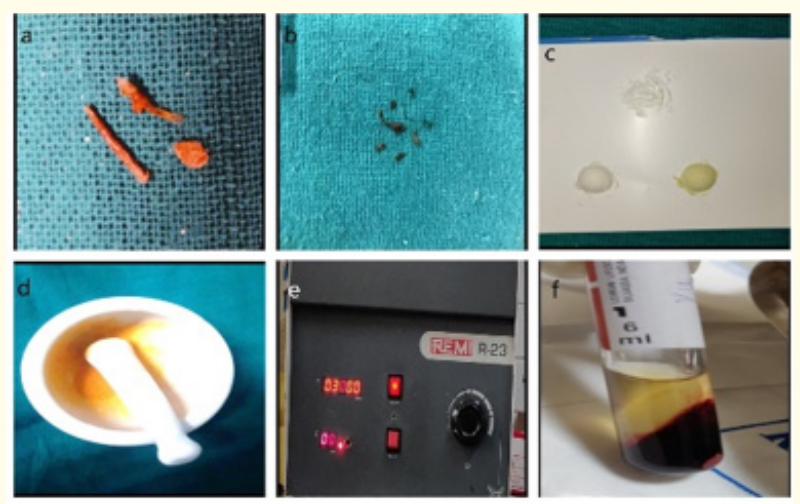

Figure 2: $(a, b)$ : Removal of gutta percha form maxillary central incisors. 2(c): Fabrication of the 3 Mix Triple Antibiotic Paste:

1:1:1 for Permanent left maxillary central incisor. 2(d): Turmeric as an intra-canal medicament for permanent right maxillary incisor. 2(e): Centrifuge-3000 rpm. $10 \mathrm{~min}$ 2(f): Tri-layered separation of plasma components.

Citation: Sabherwal., et al. "Regenerative Endodontic Procedure as a Retreatment Alternative for Permanent Maxillary Central Incisors with Esthetic Smile Correction". Acta Scientific Dental Sciences 5.11 (2021): 46-49. 
The Phase II of treatment comprised of initial esthetic restoration of both maxillary incisors using composite strip crowns (Frasaco strip, Navadha, India) [7]. Prior to rehabilitation of congenitally missing 22, the canine was retracted distally using a fixed segmental canine retraction approach. The anchorage was established by band placement on 26 , MBT brackets with $0.022 * 0.028$ slot were bonded onto $23,24,25$; ligation was done with 0.010 " figure of eight ligature wire $(24,25,26)$. This was followed by application of closed coil spring for canine retraction distally. The alignment and levelling of the teeth was achieved using sequential wire change -Nickel Titanium 0.016", stainless steel $0.017^{*} 0.025$ followed by SS $0.019 * 0.025$. The patient was recalled at monthly intervals until six months. After the retraction of canine was optimal, the same appliance was continued as a retainer [8].

Phase III incorporated the temporary restorative rehabilitation of 22. It was a technique sensitive procedure due to the lack of space availability (mesiodistal width $=6 \mathrm{~mm}$ ) despite the retraction of canine. A fiber-reinforced composite strip (Ribbond EverStick ${ }^{\circledR} \mathrm{C} \& \mathrm{~B}$; GC EUROPE N.V., Belgium) was bonded to the palatal aspect of 21 and 23 to which an acrylic pontic of mandibular central incisor was reshaped and bonded using light cure flowable composite resin (Aelite Flo LV, Bisco, IL, USA) [9]. Oral hygiene instructions and preventive dietary care was reinforced at each visit in addition to the clinical and radiographic follow-ups. The patient demonstrated complete resolution of clinical signs and symptoms at 4 weeks post treatment. The radiographic assessment documented progressive increase in root length and thickness by continued maturation of the apices at 24 months follow-up resembling Chen type I [11], type III (21) healing response [10]. The patient and parent were counselled regarding future outcomes and management options.
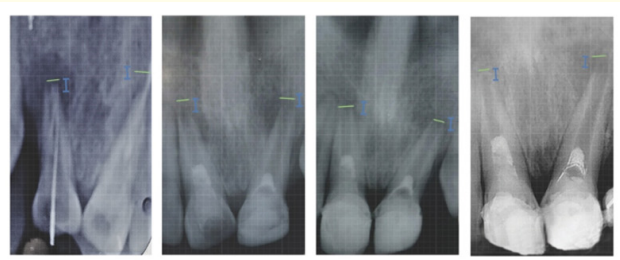

\footnotetext{
a. Pre operative Intraoral periapical radiograph

b. Post operative follow up intra oral periapical radiograph at 6 months follow up

c. Postoperative periapical radiograph at 13 month follow up

d. Postoperative follow up radiograph at 24 months follow up
}

Figure 3: Post operative buccal view.

\section{Discussion}

Regenerative endodontic procedures facilitates the reestablishment of the pulp-dentin complex. Unlike the conventional apexification procedure and apical plug technique using MTA, it does not weaken the tooth structure while promoting normal maturation and thickening of roots [11].

Regenerative endodontic procedures would ideally aim for a complete revitalization of the teeth (tertiary goal), whilst documented as a case report by Torbinejad., et al. this may not be achieved frequently during routine clinical procedures. The desirable and clinically practical outcome (secondary goal) may be the increase in root length and/ or thickness [12,13]. Chen., et al. described five secondary healing outcomes for necrotic teeth with apical periodontitis that are commonly observed as a result of regenerative endodontic procedures - type 1 , continued root maturation and increase in thickness; type 2, root apex becomes close and blunt with no increase in length; type 3 , continued increase in root length with open apices; type 4, calcification into the canal space; type 5 , formation of a hard barrier between the coronal seal and MTA plug. The healing outcome type 1 was seen for 11 and Type III with 21 at 24 months follow-up [10].

For the success of the technique, the role of intracanal medicaments and irrigation protocols are crucial. The use of intracanal medicaments such as triple antibiotic paste and calcium hydroxide is a proven choice in the REP protocol which may promote re-integration of the pulp-dentin complex. The use of medicament up to 4 weeks does not detrimentally affect the root dentin strength. The application of the triple antibiotic paste may be potent in sterilizing lesions in root canal and periodontal regions that may hasten the healing response [4]. Turmeric is a medicinal phenolic compound with antioxidant, anti-inflammatory, antispasmodic and anti-cancer effects. It is a proven effective endodontic intra-canal medicament with bactericidal effects including efficacy against endodontic pathogen E. faecalis with additional bactericidal effect on carious dentin and periodontal pockets [5]. In the present study, triple antibiotic paste and turmeric were employed as intracanal medicaments leading to successful resolution of signs and symptoms as discussed above. However, further in-vivo assessment of turmeric and its complex biologic interaction in REP would lay more clarity on its long-term outcomes. 


\section{Conclusion}

Regenerative Endodontic Procedures proves to be a promising management option for retreatment cases of root canal failure in the young adolescent. This paves way to future well-documented clinical trials assessing the nature of healing outcomes in this scenario. The literature documented thus far has studied the use of in vivo stem cells that reconstituted the necrosed tissues. Attempts to harness in vitro stem cells in artificially simulated and pathogen free lab environment may be the future revolution pending further trials [14].

\section{Conflict of Interest}

The manuscript has no conflict of interests.

\section{Bibliography}

1. Murray, Peter E., et al. "Regenerative endodontics: a review of current status and a call for action". Journal of Endodontics 33.4 (2007): 377-390.

2. Nygaard-Ostby B and Hjortdal O. "Tissue formation in the root canal following pulp removal". Scandinavian Journal of Dental Research 79 (1971): 333-349.

3. Windley W., et al. "Disinfection of immature teeth with a triple antibiotic paste". Journal of Endodontics 31.6 (2005): 439-443.

4. Hoshino E., et al. "In-vitro antibacterial susceptibility of bacteria taken from infected root dentine to a mixture of ciprofloxacin, metronidazole and minocycline". International Endodontic Journal 29.2 (1996): 125-130.

5. Prabhakar A., et al. "Comparison of Antibacterial Efficacy of Calcium Hydroxide Paste, 2\% Chlorhexidine Gel and Turmeric Extract as an Intracanal Medicament and their Effect on Microhardness of Root Dentin: An in vitro Study". International Journal of Clinical Pediatric Dentistry 6.3 (2013): 171-177.

6. Kobayashi M., et al. "A proposed protocol for the standardized preparation of PRF membranes for clinical use". Biologicals 40.5 (2012): 323-329.

7. Karaali Abdullah Emre., et al. "Composite Resin Strip Crowns used for Rehabilitation of Maxillary Central Incisor: Two Cases Report". Journal of Dental and Oral Health (2016).
8. Mrudul Vaidya., et al. "Orthodontic treatment of a crowding case using segmental arch technique: A case report". International Journal of Medical and Dental Case Reports 5.1 (2018): 1-3.

9. Pankratz V., et al. "Anterior fiber-reinforced ribbon composite resin bridge-A case report". Clinical Case Report 6.10 (2018): 1941-1946.

10. Chen MY., et al. "Responses of immature permanent teeth with infected necrotic pulp tissue and apical periodontitis/abscess to revascularization procedures". International Endodontic Journal 45.3 (2012): 294-305.

11. Iwaya SI., et al. "Revascularization of an immature permanent tooth with apical periodontitis and sinus tract". Dental Traumatology 17 (2001): 185-187.

12. Lin LM and Kahler B. "A review of regenerative endodontics: current protocols and future directions". Journal of Istanbul University Faculty of Dentistry 51 (2017): S41-S51.

13. Torabinejad M., et al. "Regenerative endodontic treatment or mineral trioxide aggregate apical plug in teeth with necrotic pulps and open apices: a systematic review and meta-analysis". Journal of Endodontics 43.11 (2017): 1806-1820.

14. Jang JH., et al. "Pulp regeneration with hemostatic matrices as a scaffold in an immature tooth minipig model". Scientific Reports 10.1 (2020): 12536.

\section{Volume 5 Issue 11 November 2021 (C)All rights are reserved by Sabherwal., et al.}

